13

\title{
Особенности разработки электронно-оптических систем для импульсных терагерцовых ламп бегущей волны (Обзор)
}

\author{
(C) А.А. Бурцев, ${ }^{1,2}$ Ю.А. Григорьев, ${ }^{1}$ А.В. Данилушкин, ${ }^{1,2}$ И.А. Навроцкий, ${ }^{1}$ А.А. Павлов, ${ }^{3}$ К.В. Шумихин ${ }^{1}$ \\ ${ }^{1}$ Акционерное общество Научно-производственное предприятие „Алмаз“, \\ 410033 Саратов, Россия \\ ${ }^{2}$ Саратовский государственный технический университет им. Ю.А. Гагарина, \\ 410054 Саратов, Россия \\ ${ }^{3}$ Институт нанотехнологий микроэлектроники РАН, \\ 115487 Москва, Россия \\ e-mail: antbourtsew@gmail.com
}

(Поступило в Редакцию 11 мая 2017 г. В окончательной редакции 23 августа 2017 г.)

\begin{abstract}
Представлен анализ современного состояния и разработки импульсных усилительных ламп бегущей волны терагерцового излучения, работающих в диапазоне частот от $200 \mathrm{GHz}$ и выше, а также перспектив развития принципов создания электронно-оптических и магнитных систем. Обсуждена возможность применения автоэмиссионных катодов на основе углеродных нанотрубок для конструкции электронно-оптической системы с компрессией ленточного пучка. Представлено численное моделирование автоэмиссионной электронной пушки, формирующей ленточный электронный поток для ламп бегущей волны терагерцового диапазона.
\end{abstract}

DOI: $10.21883 /$ JTF.2018.03.45608.2331

Современные СВЧ усилители, используемые для широкополосной и космической средств связи, разрабатываются с применением базовых технологий вакуумной электроники. В последние годы в области вакуумной СВЧ электроники особый интерес привлекает проблема освоения терагерцового диапазона, решение которой позволит создать усилители терагерцового диапазона, которые могут найти широкое применение в таких областях, как информационно-коммуникационные системы нового поколения, радиоастрономия дальних космических объектов, спектроскопия, диагностика плазмы, бесконтактный контроль качества производства. Этот диапазон является неосвоенной частью электромагнитного спектра, поскольку в этом диапазоне резко падает мощность как вакуумных, так и твердотельных источников. Наиболее высокие мощности потенциально способны обеспечить гироприборы, оротроны, лазеры на свободных электронах и другие приборы. Различными научными коллективами были предложены миниатюрные аналоги классических приборов ЛБВ, ЛОВ, отражательных клистронов. В начале 2000 гг. Агентство по перспективным оборонным исследовательским проектам Министерства обороны CША (DARPA) провело открытый конкурс по стимулированию разработок ведущих американских фирм компактных вакуумных приборов коротковолновой части миллиметрового диапазона с выходной мощностью порядка десятков ватт и более. Основное внимание тогда и сегодня уделяется созданию приборов на частоты 220, 460, 670, 850 и $1030 \mathrm{GHz}$. Компактные усилители средней мощности могут быть реализованы на основе миниатюризированных приборов вакуумной СВЧ электроники, таких как ЛБВ, клистрон бегущей волны. По оценкам отечественных и зарубежных исследователей, наиболее приемлемыми к приме- нению в качестве замедляющих систем (3С) для ЛБВ терагерцового диапазона являются 3С следующих типов: петляющий волновод, плоская гребенка, встречные штыри и меандр различной конфигурации. Во многих работах обсуждаются перспективы создания многосекционных ЛБВ терагерцового диапазона с ленточными или многолучевыми электронными потоками. Для реализации таких конструкций традиционные технологии, используемые для изготовления узлов приборов вакуумной СВЧ электроники сантиметрового и миллиметрового диапазонов, технологически трудно реализуемы вследствие роста высокочастотных потерь, трудностей прохождения электронных пучков через микроразмерные пролетные каналы ЗС и снижения эффективности взаимодействия электронного потока с электромагнитным полем (КПД $\approx 1-2 \%$ ). В научных подразделениях компаний Northrop Grumman, Teledyne Scientific, Teledyne MEC и Калифорнийского университета Дэвис ведутся разработки в рамках совместной программы по созданию ЛБВ усилителя на $220 \mathrm{GHz}$ и более на термокатодах. Изготовление волноведущих и замедляющих систем выполняется на основе МЭМС технологий с уровнем допусков 3-5 $\mu \mathrm{m}$ и шероховатостью поверхности не более $30 \mathrm{~nm}$. Также для этих же целей исследуются возможности применения 3D-печати. По утверждению разработчиков компаний Northrop Grumman, Teledyne, L-3 Communications and Power Industries, Beam-Wave Rescore и др. традиционная электроискровая технология изготовления миниатюрных узлов в диапазоне $220 \mathrm{GHz}$ и выше непригодна, так как не может обеспечить уровень чистоты внутренней поверхности щелей, необходимой для сведения к минимуму омических потерь 3С.

На проводимой ежегодно международной конференции IEEE по вакуумной электронике (IVEC) подробно 


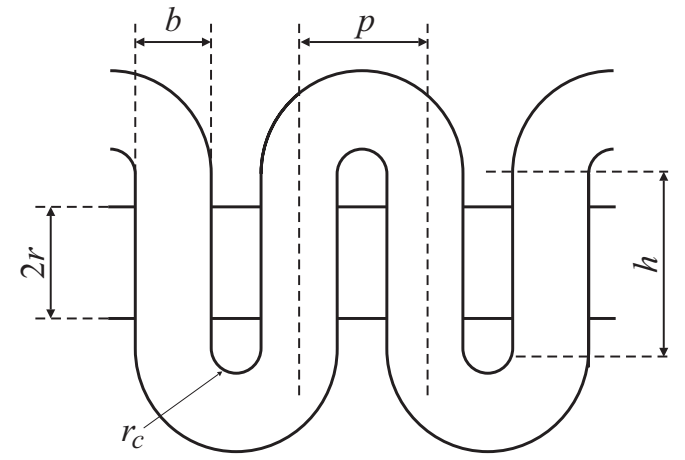

Рис. 1. Схема петляющего волновода. $b-$ продольная толщина стенки, $p-$ период структуры, $r_{c}-$ внутренний радиус волновода, $h-$ высота прямой части, $r$ - радиус канала.

представлены результаты исследования и разработки в рамках программы HiFIVE макетов вакуумных и узлов СВЧ усилителей терагерцового диапазона ведущими зарубежными фирмами. В работе [1] представлена система типа петляющий волновод (рис. 1), спроектированной для работы в диапазоне $220 \mathrm{GHz}$ компанией Northrop Grumman. Разработаны две основных технологии изготовления: LIGA и DRIE - технология глубокого реактивно-ионного травления. В работе ЗС изготавливается вместе с входным и выходным волноводами в одном монолитном блоке металла. Это позволяет значительно упростить сборку приборов подобного типа и решает проблему теплоотвода от элементов ЗС. Указано, что технология ионного травления позволяет получать допуски изготовления до $\pm 0.5 \mu \mathrm{m}$. Одной из первых экспериментальных работ в этом направлении является разработка вакуумного электронного генератора на $0.65 \mathrm{THz}$ на основе петляющего волновода [2]. Электродинамическая часть в экспериментальных приборах была сделана с помощью глубокого реактивного травления материала ионным лучом (DRIE). Несколько генераторов были сделаны и работают на частотах между 0.605 и $0.675 \mathrm{THz}$. ВЧ мощность достигала $98 \mathrm{~mW}$ с максимумом $50 \mathrm{~mW}$, измеренным за окном энергии. Эти измерения были выполнены в импульсном режиме с коэффициентом заполнения до 3\%. Недавно компания продемонстрировала компактный электровакуумный усилитель с выходной мощностью свыше $50 \mathrm{~mW}$ в полосе $835-842 \mathrm{GHz}$ и $39.4 \mathrm{~mW}$ на частоте $850 \mathrm{GHz}$ [3]. Его коэффициент усиления в режиме малого сигнала составил $26 \mathrm{~dB}$ в рабочей полосе $11 \mathrm{GHz}$. К конструктивным особенностям усилителя относятся: ЗС с петляющим волноводом, термокатод с большой плотностью тока, фокусирующая магнитная система на основе соленоида и одноступенчатый коллектор. Для фокусировки пучка использовали магнитную систему из $\mathrm{NdFeB}$, обеспечившую на участке длиной $2.5 \mathrm{~cm}$ аксиальную магнитную индукцию $0.9 \mathrm{~T}$. В эксперименте с коэффициентом заполнения импульса напряжения до $1 \%$ наблюдалось токооседание пучка на 3С порядка 60\%. Ток катода составил $2.81 \mathrm{~mA}$ при уско- ряющем напряжении $11.45 \mathrm{kV}$. Необходимо отметить, что в экспериментах наблюдались небольшое падение тока пучка и снижение выходной мощности, когда коэффициент заполнения увеличился от 0.1 до $1 \%$. Сделан вывод о том, что работа усилителя при более высоком коэффициенте заполнения требует еще более точной установки электронной пушки относительно пролетного канала. Следующая запланированная по программе DARPA фаза разработок этой компанией - это разработка усилителя на $0.67 \mathrm{THz}$ с большей долговечностью, разработка усилителя на $1.03 \mathrm{THz}$ на основе петляющего волновода, созданного по технологии DRIE.

Ориентируясь на разработку усилителей мощности mm-диапазона для новых систем связи и РЛС, исследовательская лаборатория NRL исследовала ЛБВ c 3С на основе петляющего волновода. В настоящее время лаборатория разрабатывает методы изготовления замедляющей системы малых размеров, опираясь на многослойную технологию UV-LIGA для обработки меди. Так, в работе [4] по данной технологии разрабатывается усилитель бегущей волны на $231-233 \mathrm{GHz}$. Основные цели разработки: более $140 \mathrm{~W}$ выходной мощности, $15 \mathrm{GHz}$ ширина полосы. Фокусировка пучка с током $124 \mathrm{~mA}$ осуществляется плоскопараллельным магнитным полем 0.66 Т длиной $1.3 \mathrm{~cm}$ при ускоряющем напряжении $20 \mathrm{kV}$, причем диаметр пролетного канала составляет $200 \mu \mathrm{m}$. Используется низкопервеансная электронная пушка с нарастающим магнитным полем и с компрессией электронного пучка. Отмечено, что смещение пролетного канала относительно центра поперечной плоскости 3С является важным и существенно влияющим на параметр затухания S21.

Эффективность работы усилительной ЛБВ терагерцового диапазона связана с применением многосекционных 3С, а также возможности рекуперации электронного пучка. Так, в работе [5] приведены результаты использования принципа многосекционной ЛБВ, где разработана трехсекционная 3С типа „петляющий волновод“, в которой происходит последовательное усиление СВЧ сигнала, причем все секции расположены планарно. В каждой секции взаимодействие волны происходит с отдельным электронным потоком, т.е. система сопровождения электронного потока фактически формирует три пучка. При этом ток электронного пучка составляет $100 \mathrm{~mA}$ электронного потока при ускоряющем напряжении около $20 \mathrm{kV}$. Учитывая величину выходной мощности, можно заключить, что электронный КПД рассмотренного в работе прибора может составить примерно 5\%. На конференции IVEC 2017 представлен первый, как утверждают авторы, экспериментальный образец усилителя в диапазоне $213-235 \mathrm{GHz}$ на основе ЛБВ с двухсекционным петляющим волноводом [6]. Максимальная выходная мощность усилителя составила $176 \mathrm{~W}$ при коэффициенте заполнения $50 \%$ и длительности импульса $25 \mu \mathrm{s}$. При использовании в конструкции многоступенчатого коллектора КПД лампы составил 11\%. Область применения 


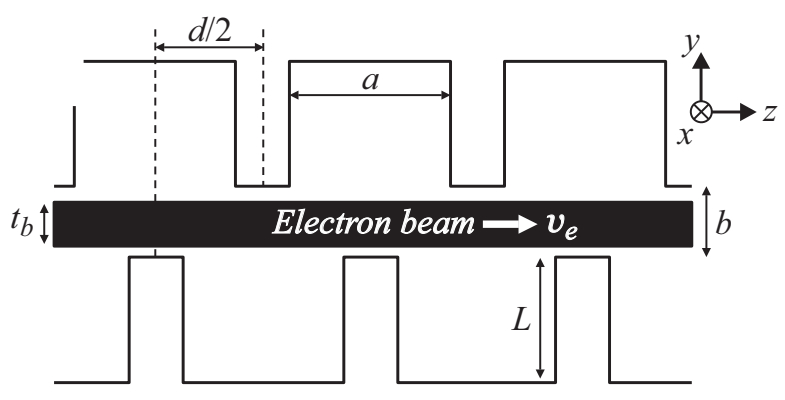

Pис. 2. Схема 3 С типа сдвоенная гребенка. $t_{b}-$ толщина ленточного пучка, $L, d / 2, a, b-$ геометрические параметры гребенки.

данного усилителя - предположительно новые системы радаров с высоким разрешением.

Для обеспечения эффективного взаимодействия протяженного ленточного электронного пучка с замедленной электромагнитной волной также рассматриваются приборы на основе ЗС типа плоских гребенок. Хотя подобные ЗС были разработаны достаточно давно, в связи с переходом в более коротковолновый диапазон интерес к ним вырос [7-11]. Также это обусловлено появлением новых технологий, позволяющих изготавливать системы с размерами, необходимыми для работы в указанных диапазонах частот и малыми разбросами, а также разработкой новых методов численного моделирования распространения электромагнитных волн и их взаимодействия с электронными потоками в ЗС со сложной геометрией. В Калифорнийском университете разработки были направлены на создание ЛБВ-усилителя $220 \mathrm{GHz}$ выходной мощностью более $50 \mathrm{~W}$ с коэффициентом усиления $30 \mathrm{~dB}$. В [9] была предложена 3С в виде двойной гребенки с противофазным расположением штырей, приведенной на рис. 2. В электронно-оптической системе предлагается использовать ленточный пучок с током $250 \mathrm{~mA}$ с компрессией 7 при ускоряющем напряжении $20 \mathrm{kV}$. Последующие исследования [10-11] были направлены на отработку технологий изготовления функциональных узлов макетов прибора, холодных измерений параметров 3С, измерений в малосигнальном режиме, а также на экспериментальные исследования фокусировки и токопрохождения ленточных пучков в пролетном канале.

Основной проблемой при разработке вакуумных приборов терагерцового диапазона является необходимость использовать электронные пучки с высокой плотностью тока ввиду уменьшения поперечных размеров пролетных каналов в ЗС. В большинстве случаев в пространстве взаимодействия требуется высокая плотность тока, достигающая значений $500 \mathrm{~A} / \mathrm{cm}^{2}$, что является трудно осуществимым для современных катодов. Поэтому привлекли внимание те конструкции, в которых используются компрессионные электронно-оптические системы (ЭОС) с ленточным или цилиндрическим пучком. Известно из [12], что плотность тока в электронном пучке связана с частотой бегущей и стоячей волн выражением

$$
P_{t w}=24 N(1 / f)^{8 / 3} U_{0}^{13 / 6} j^{4 / 3},
$$

где $P_{t w}$ - пиковая мощность, $W, N-$ аспектное соотношение ленточного пучка или количество парциальных цилиндрических пучков, $f-$ частота, $\mathrm{GHz}, U_{0}-$ напряжение пучка, $\mathrm{kV}, j$ - плотность тока в $\mathrm{A} / \mathrm{cm}^{2}$. Это означает, что для получения умеренных значений выходной мощности прибора требуется создание ЭОС с магнитным сопровождением и с высокой плотностью тока в поперечным сечении пучка, достигающей значений более $100 \mathrm{~A} / \mathrm{cm}^{2}$. Естественно, что в (1) не учитываются сложности формирования и фокусировки протяженного пучка электронов с ростом его плотности тока при соответствующем уменьшении пролетного канала. За последние 10 лет появилось множество работ, посвященных разработкам и исследованию эффективных скандатных термокатодов с высокой плотностью тока. Можно выделить одну из таких разработок, опубликованную в ряде работ $[13,14]$, представленных коллективом из Калифорнийского университета. Так, работе [13] исследуется катод из нанокомпозита вольфрама с добавками $\mathrm{Sc}_{2} \mathrm{O}_{3}$ для $220 \mathrm{GHz}$ ЛБВ. Электронная пушка рассчитана на плотность постоянного тока $10 \mathrm{~A} / \mathrm{cm}^{2}$ при температуре $1120^{\circ} \mathrm{C}$ с наработкой более $2000 \mathrm{~h}$. Для другой пушки с уменьшенным зазором катод-фокусирующий электрод до $30 \mu \mathrm{m}$ получена плотность постоянного тока $45 \mathrm{~A} / \mathrm{cm}^{2}$. Импульсный ток с плотностью от $56 \mathrm{~A} / \mathrm{cm}^{2}$ при $960^{\circ} \mathrm{C}$ и до $104 \mathrm{~A} / \mathrm{cm}^{2}$ при $1040^{\circ} \mathrm{C}$ получен на следующем макете пушки с зазором в $70 \mu \mathrm{m}$ и напряжением $4 \mathrm{kV}$. Для долговременной работы терагерцового прибора также могут использоваться металлосплавные термокатоды из сплава $\mathrm{Ir}-\mathrm{Ce}$ [15].

ЭОС с компрессией электронного потока и соответственно с магнито-экранированным катодом позволяют получить достаточно большие плотности тока в пучке при меньшей токовой нагрузке на катод и с меньшим значением рассчитанного магнитного поля. Для создания электронной пушки, формирующей сходящийся ленточный пучок, существует конструкция, включающая цилиндрический катод и две пары фокусирующих электродов [16]. Для придания пучку необходимого угла сходимости применяется пара основных фокусирующих электродов, а также боковые электроды, ограничивающих расширение пучка по ширине. Эти боковые электроды должны создавать вдоль несходящихся краев потока такое же распределение потенциала, что и основные электроды. Принцип компрессии эллиптического пучка и получение за анодным отверстием в кроссовере ленточного пучка применен для разработки ЛБВ с выходной мощностью $50 \mathrm{~W}$, работающей на частоте $220 \mathrm{GHz}$, где рассчитан вариант пушки с ленточным пучком с током $257 \mathrm{~mA}$ при ускоряющем напряжении $20 \mathrm{kV}$, формируемом с эллиптического катода за счет фокусирующего электрода сложной формы [17]. Линейная компрессия пучка порядка 7 осуществляется с катода при средней 


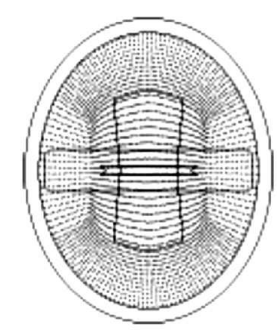

$Z=0 \mathrm{~mm}$

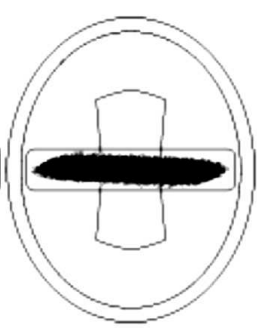

$Z=12 \mathrm{~mm}$

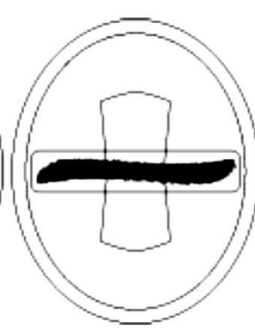

$Z=22 \mathrm{~mm}$

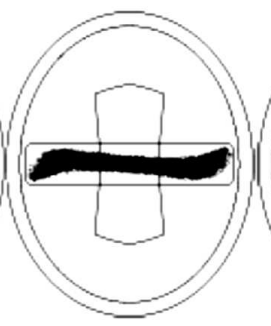

$Z=32 \mathrm{~mm}$

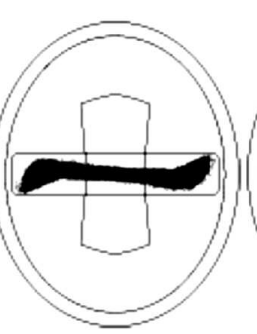

$Z=42 \mathrm{~mm}$

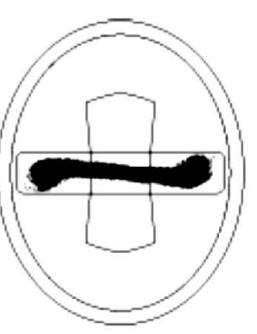

$Z=52 \mathrm{~mm}$

Рис. 3. Расчет фокусировки в программе MICHELLE компрессионного ленточного пучка в пушке с эллиптическим катодом на длину взаимодействия $52 \mathrm{~mm}[17]$.
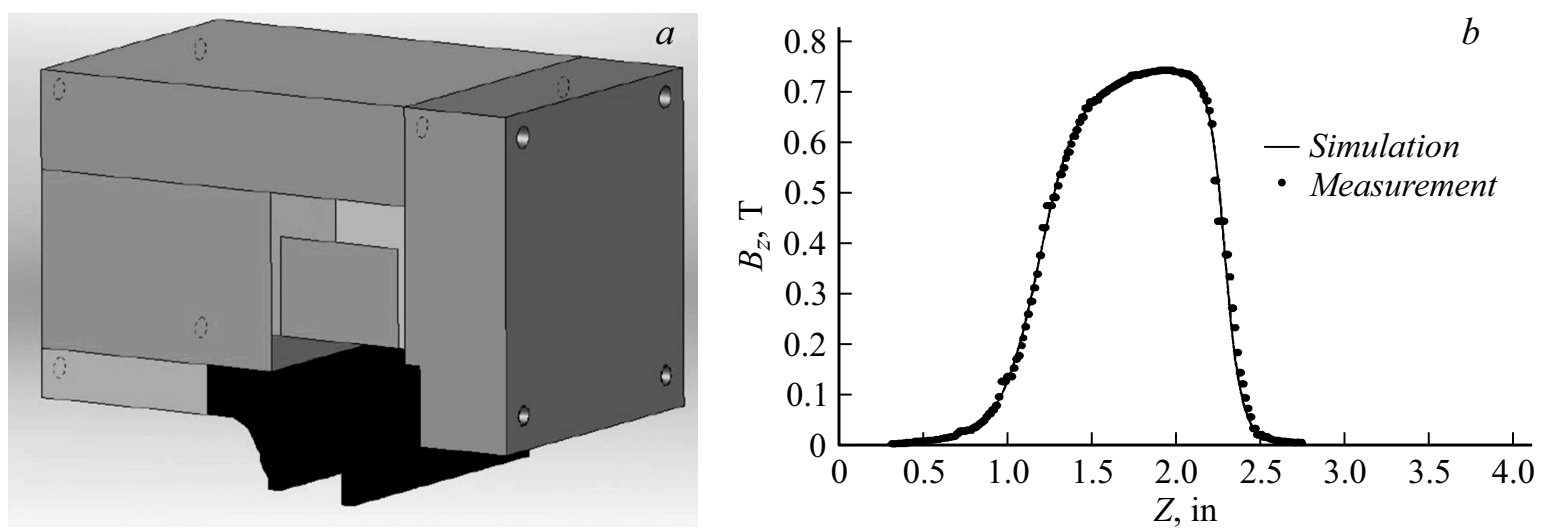

Рис. 4. Магнитная система для ленточного со сходимостью пучка с рабочей длиной $2.5 \mathrm{~cm}$ с индукцией $0.75 \mathrm{~T}$ [20].

плотности тока на катоде $40 \mathrm{~A} / \mathrm{cm}^{2}$ (рис. 3). В работе [18] проведен расчет электронной пушки для ЛБВ $220 \mathrm{GHz}$, в которой формируется ленточный электронный пучок с током $80 \mathrm{~mA}$ и напряжением $25 \mathrm{kV}$. Пучок формируется с поверхности цилиндрического катода радиусом $0.35 \mathrm{~mm}$, т.е. плотность тока на катоде составляет $20 \mathrm{~A} / \mathrm{cm}^{2}$. В пушке применена компрессия электронного пучка в соотношении 7:4 и 7:1 по осям $x$ и $y$ соответственно, и в результате формируется пучок сечением $0.4 \times 0.1 \mathrm{~mm}$. Для более коротковолновых приборов в работе [19] приведены результаты моделирования высококомпрессионной ЭОС с плотностью тока $750 \mathrm{~A} / \mathrm{cm}^{2}$ ленточного пучка в пролетном канале высотой $150 \mu \mathrm{m}$ и общим током $400 \mathrm{~mA}$. Линейная компрессия пучка составила 25. Экспериментальные исследования подобных пушек, но с током $250 \mathrm{~mA}$ подтвердили результаты моделирования и получено стопроцентное токопрохождение через анодное отверстие. Из рис. 3 видно, что для систем с протяженным ленточным потоком сопутствует проблема его деформации в магнитном поле по мере увеличения длины пролетного канала, что может привести к токооседанию в микроразмерном канале. Особенно это актуально для пушек термокатодами. Одним из ограничений использования термокатодов является влияние фазового объема и начальных тепловых скоростей электронов на фокусировку электронных потоков в зависимости от температуры катода. Например, для ме- таллопористых термокатодов средняя тепловая скорость соответствует ускоряющему напряжению порядка $0.7 \mathrm{~V}$. Это приводит к сушественному расширению пучка в канале и перераспределению плотности тока в нем.

Эффективная магнитная фокусировка электронных пучков с большой плотностью тока существенна для успешной разработки вакуумных приборов терагерцового диапазона. К основным проблемам можно отнести создание систем с повышенной однородностью магнитного поля и материалами, обеспечивающими относительно высокие значения полей (0.6-1.0T). Макеты с ленточным пучком, где длина взаимодействия порядка $2-3 \mathrm{~cm}$ имели магнитные системы с большими габаритами (рис. 4). Для компактности магнитных систем могут применяться магнитные периодические или реверсные системы. Приближенно распределение магнитного поля в них записывается следующими уравнениями [21]:

$$
\begin{gathered}
B_{y, m}(y, z) \approx-B_{0} \sinh \left(k_{m} y\right) \cos \left(k_{m} z\right), \\
B_{z, m}(y, z) \approx B_{0} \cosh \left(k_{m} y\right) \sin \left(k_{m} z\right),
\end{gathered}
$$

где $B_{0}-$ амплитуда магнитного поля, $k_{m}=2 \pi / \lambda_{m}$ и $\lambda_{m}$ - период магнитной системы. Так, в работах сотрудников Калифорнийского университета Дэвис [22,23] для обеспечения пропускания электронного пучка с высокой плотностью тока предлагается периодическая магнитная система (рис. 5). Такая система проектируется в сочетании с квадрупольным магнитом для улучшения коэф- 

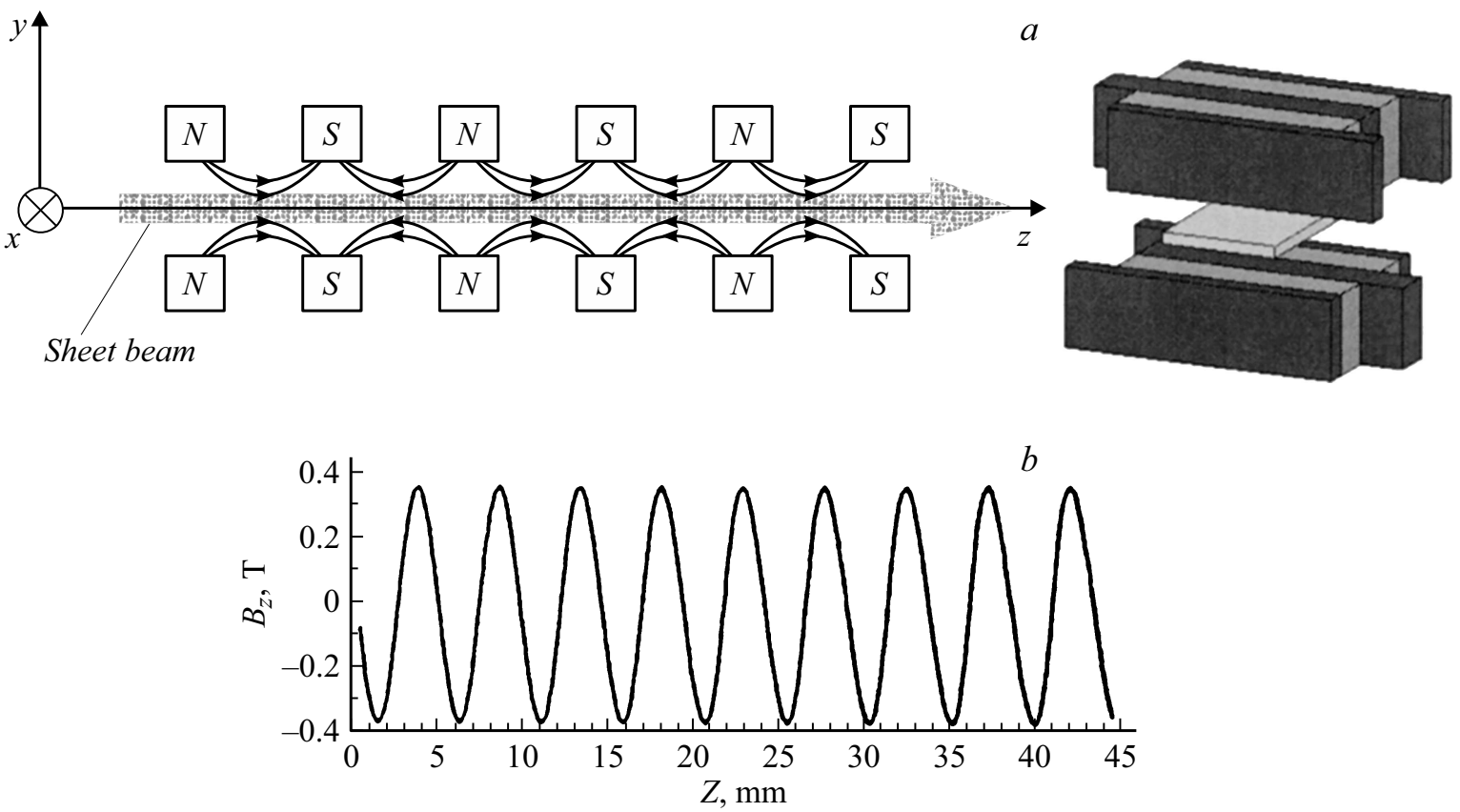

Рис. 5. Схематическое изображение плоскопараллельной магнитной периодической системы для фокусировки ленточного пучка и график распределения поля $B_{z}$ на длину $45 \mathrm{~mm}$, представленной в работе [23].
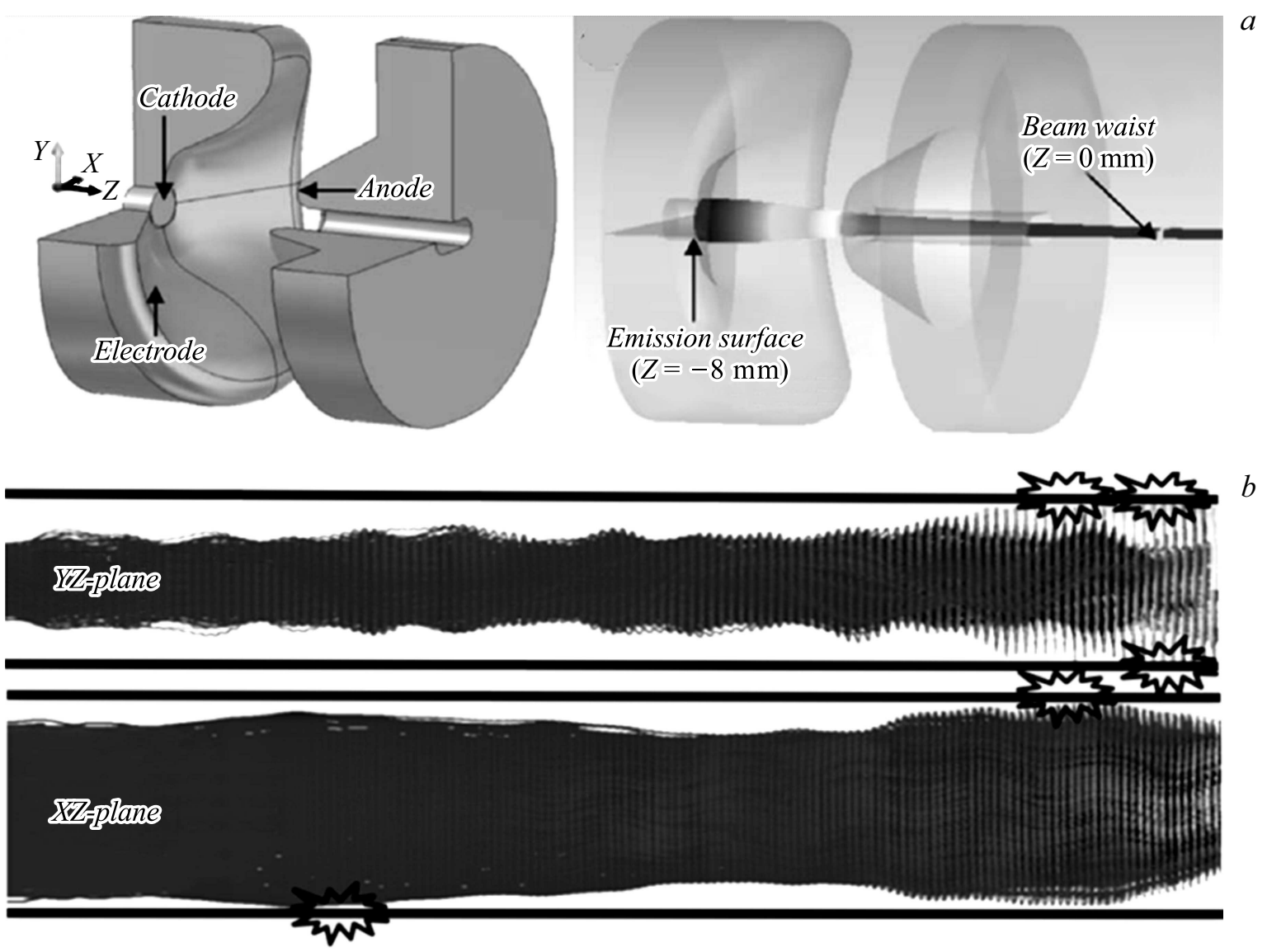

Рис. 6. Электронная пушка с компрессией ленточного пучка (a) и группировка его в ЭОС в $Y Z$ и $X Z$ плоскостях $(b)$. 

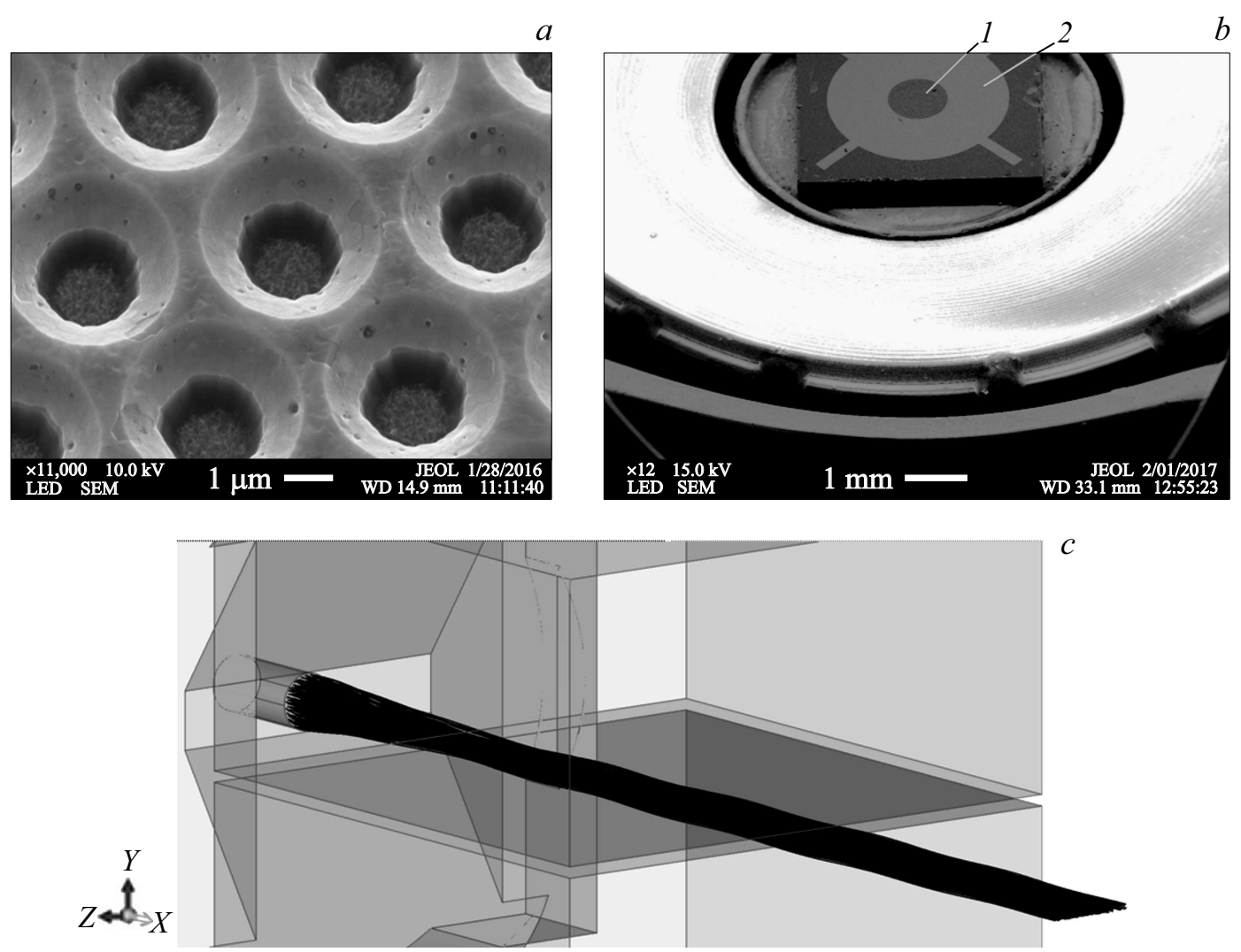

Рис. 7. СЭМ изображение АЭК с УНТ вид сверху $(a)$ и электронной пушки $(1-$ эмиттер, 2 - проводящий слой для подключения тянущей сетки) [36] (b), фокусировка ленточного пучка с плоской автоэмиссионной катодно-сеточной структуры из УНТ (c).

фициента пропускания и уменьшения угла деформации ленточного пучка. Магнитная периодическая система обеспечивает фокусировку пучка вдоль оси $Z$, а квадрупольная ее часть осуществляет удержание пучка в поперечном направлении. Показано с помощью анализа, что магнитная система обеспечивает фокусировку с учетом начального поперечного разброса тепловых скоростей и увеличения плотности тока, вызванного группировкой пучка (рис. 6). Эта фокусирующая система используется для транспортировки ленточного пучка с параметрами: $19 \mathrm{kV}, 0.15 \mathrm{~A}$, компрессией 8.5 , с поперечным сечением эллиптической формы пучка $0.60 \times 0.07 \mathrm{~mm}$. Плотность тока в пучке превышает $400 \mathrm{~A} / \mathrm{cm}^{2}$, в пролетном канале двухсекционного петляющего волновода длиной $45 \mathrm{~mm}$ (для $263 \mathrm{GHz}$ ), размер которого составляет $0.7 \times 0.12 \mathrm{~mm}$. С применением такой магнитной системы коэффициент токопрохождения прогнозируется на уровне $95 \%$.

Заменой термокатодов в терагерцовых ЭОС могут стать катоды на основе автоэмиссии. Процесс внедрения автоэмиссионных катодов (АЭК) в мощные СВЧ приборы обусловлен технологическими особенностями применяемых материалов и сложностями получения геометрически воспроизводимых многоострийных катодных и катодно-сеточных структур с наноразмерными вершинами острий. Сложности возрастают при про- ектировании автоэмиссионных электронных пушек с компрессией по плотности тока в поперечном сечении формируемого электронного пучка. Интенсивные исследования за последние 20 лет по совершенствованию технологий изготовления катодных матриц Спиндта [24], эмиттеров на основе углеродных нанотрубок (УНТ), алмазоподобных пленок [25-29] привели к созданию экспериментальных образцов электронных пушек для вакуумных усилителей О-типа. В 2009 г. фирмой L-3 Communication исследованы выходные характеристики ЛБВО С-диапазона [30]. С катода диаметром $1 \mathrm{~mm}$ получен ток $121 \mathrm{~mA}$, что соответствует плотности тока $15.4 \mathrm{~A} / \mathrm{cm}^{2}$. При напряжении замедляющей структуры $3500 \mathrm{~V}$ на частоте $5 \mathrm{GHz}$ выходная мощность составила $\approx 100$ W. В 2013 г. была представлена ЛБВО Х/Ки диапазона с катодом Спиндта [31]. При токе катода $50 \mathrm{~mA}$ на частоте $10.6 \mathrm{GHz}$ при усилении $13.5 \mathrm{~dB}$ выходная мощность не превышала $10 \mathrm{~W}$. Одной из первых попыток создать электронную оптику для терагерцового прибора на основе матричного АЭК представлена в работе [32]. Катодно-сеточная структура представляла островки из массива многостенных УНТ, выращенных на кремниевой подложке и окруженных тонкопленочной сеткой. Пушка погружена в постоянное магнитное поле $0.9 \mathrm{~T}$, ток до $4 \mathrm{~mA}$, напряжение анода $10 \mathrm{kV}$, микропервеанс $0.0014 \mathrm{~A} / \mathrm{V}^{3 / 2}$. Максимальная плотность тока с такого 
катода в постоянном режиме составила $2 \mathrm{~A} / \mathrm{cm}^{2}$. В работе [33] предложена и разработана триодная конструкция электронной пушки с УНТ с микровакуумным зазором для создания СВЧ усилителя, полностью интегрированной на одной подложке с помощью МЭМС технологий. Такая электронная пушка по результатам исследований с матрицей $3 \times 3$ из УНТ с размером $9 \mu \mathrm{m}$ работала в непрерывном режиме с плотностью автоэмиссионного тока более $1 \mathrm{~A} / \mathrm{cm}^{2}$. Из-за ограниченной эмиссии автокатодов сформировать пучки для ЭОС терагерцовых приборов оказывается возможным путем использования компрессионной электронной оптики. Автоэмиссионные свойства и высокоаспектная структура УНТ определяют потенциальные возможности для использования их в качестве АЭК применительно к приборам вакуумной СВЧ электроники. Преимущество процессов CVD в сочетании с последовательными технологическими процессами микроэлектроники позволяют сформировать триодную автоэмиссионную катодно-сеточную структуру с вертикальным расположением нанотрубок (рис. 7,a), обеспечивая при этом требуемое расчетное расстояние катод-сетка, для создания на поверхности УНТ номинальных значений напряженности поля. Для исследования ВАХ катодно-сеточная структура из УНТ на кремниевой подложке устанавливалась с помощью пайки в блоке электродов (рис. 7,b) для подачи импульсного напряжения на тянущую сетку. Относительно малое напряжение управления током катода (несколько вольт и десятков вольт) в отличие от высоковольтных острийных автокатодов [34] приводит к снижению процессов ионизации в узле и электротермического распыления острия, что способствует более долговечной и стабильной работе СВЧ приборов [35]. На рис. 7,c представлено моделирование ленточного пучка в Lorentz-3EM при ускоряющем напряжении $20 \mathrm{kV}$ и током пучка $10 \mathrm{~mA}$, сфокусированного с плоской автоэмиссионной катодно-сеточной структуры из УНТ диаметром $1 \mathrm{~mm}$. Линейная компрессия обеспечивается применением фокусирующего электрода, имеющего в одной плоскости необходимый угол для сходимости пучка 10 и боковую часть, ограничивающую расширение пучка по ширине. Размеры ленточного пучка за анодным отверстием составили $0.1 \times 1 \mathrm{~mm}$. Ленточный пучок удерживается в ЭОС плоскопараллельным магнитным полем с индукцией 0.6 Т. Основным допущением в модели ЭОС является использование катода с гладкой поверхностью. Вычисленный градиент потенциала вблизи его поверхности составил $0.4 \cdot 10^{5} \mathrm{~V} / \mathrm{cm}$ при ускоряющем потенциале $20 \mathrm{kV}$, который в катоде за счет коэффициента усиления электрического поля в УНТ достигает величины, необходимой для автоэмиссии. Тянущая сетка находится под естественным потенциалом и занимает то же положение, что и эквипотенциальная поверхность в диодной пушке.

Таким образом, обобщая результаты работ, можно заключить, что применение пространственно развитых многосекционных $3 \mathrm{C}$ и ЭОС с высокой компрессией пучка дает возможность создавать приборы-усилители типа ЛБВ, работающие в терагерцовом диапазоне. Основные научные направления в области исследования эффективного усилителя терагерцового диапазона (от $200 \mathrm{GHz}$ и выше) должны быть нацелены на: усовершенствование высокоточных МЭМС технологий, в том числе 3D-печати, использование трехмерных программ компьютерного моделирования и создание методик расчета электродинамических и электронно-оптических систем терагерцового диапазона; создание микроразмерных многосекционных 3С; создание компактных ЭОС, в том числе на основе автоэлектронной эмиссии с магнитным формированием электронных потоков, с высокой компрессией ленточного пучка $10-15$, с токопрохождением 99.5-99.9\% на коллектор; создание эффективных ЛБВ терагерцового диапазона $200-220 \mathrm{GHz}$ с рекуперацией и КПД более $20 \%$.

\section{Список литературы}

[1] Tucek J.C., Basten M.A., Gallgher D.A., Kreischer K.E. // IEEE Intern. Vacuum Electron. Conf. IVEC. 2010. P. 19-20.

[2] Kreischer K.E. et al. // Intern. Conf. Infrared, Millimeter and Terahertz Waves. IRMMW-THz. 2008. P. 1-2.

[3] Tucek J.C., Basten M.A., Gallagher D.A., Kreischer K.E. // IVEC. 2014. P. 153-154.

[4] Joye C.D. et al. // IVEC. 2014. P. 219.

[5] Nguyen K., Wright E., Pershing D., Ludeking L. // IVEC. 2010. P. 23-24.

[6] Armstrong C.M. et al. // IVEC. 2017.

[7] Shin Y.M., Baig A., Gamzina D., Luhmann N.C. // IVEC. 2010. P. $185-186$.

[8] Karetnikova T.A., Ryskin N.M., Rozhnev A.G., Torgashov G.V., Shalaev P.D., Burtsev A.A. // The 42nd IEEE Int. Conf. on Plasma Sciences. 2015. DOI: 10.1109/PLASMA.2015.7179925.

[9] Shin Y.-M., Barnett L.R., Luhmann N.C. // IEEE Trans. El. Dev. 2009. Vol. 56. N 5. P. 706-712.

[10] Baig A., Gamzina D., Barchfeld R., Domier C., Barnett L.R., Luhmann N.C. // Phys. Plasm. 2012. Vol. 19. N 9. P. 093110.

[11] Field M. et al. // IVEC. 2014. P. 225-226.

[12] Levush B. et al. // Intern. Conf. on Infrared, Millimeter, and Terahertz Waves. IRMMW-THz 2009. P. 1-2.

[13] Jinfeng Zhao et al. // IVEC. 2011. P. 41-42.

[14] Jinfeng Zhao, Gamzina D., Baig A., Barnett L., Luhmann N.C., Na Li, Ji Li. // IVEC. 2012. P. 47-48.

[15] Дюбуа Б.Ч., Поливникова О.В. // Электронная техника. Сер. 1. СВЧ-техника. 2013. Вып. 4 (519). С. 187-190.

[16] Тренева С.H. АС № 105480 от 09.11.1955.

[17] Kimura T., Atkinson J., Forrest S., Grant T., Hunter T., Field M., Borwick R., Brar B. // IVEC. 2012. P. 195-196.

[18] Shi X., Wang Z., Tang X., Tang T., Gong H., Zhou Q., Bo W., Zhang Y., Duan Z., Wei Y., Gong Y., Feng J. // IEEE Trans. Plasma Sci. 2014. Vol. 42. N 12. P. 3996-4003.

[19] Atkinson J.E. et al. // IVEC. 2010. P. 97-98.

[20] Stockwell B.C. et al. // IVEC. 2010. P. 451-452.

[21] Basten M.A., Booske J.H. // J. Appl. Phys. 1999. Vol. 85. N 9. P. 6313-6322.

[22] Zheng Yuan, Gamzina D., Popovic B., Luhmann N.C. // IEEE Trans. El. Dev. 2016. Vol. 63. N 11. P. 4466-4472. 
[23] Zheng Yuan, Gamzina D., Luhmann N.C., Moran M. // IVEC. 2017.

[24] Spindt G.A. // J. Appl. Phys. 1968. Vol. 39. N 6. P. 3504-3505.

[25] Ulisse G., Ciceroni C., Brunetti F., Di Carlo A. // IEEE Trans. El. Dev. 2014. Vol. 61. N 7. P. 2558-2563.

[26] Гуляев Ю.В., Синицын Н.И., Торгашов Г.В. и др. // Радиотехн. и электрон. 2003. Т. 48. № 11. С. 1399-1406.

[27] Galperin V.A., Zhukov A.A., Pavlov A.A., Skorik S.N., Shaman Yu.P., Shamanaev A.A. // Nanotechnology Semiconductors. 2014. Vol. 48. N 13. P. 1742-1746.

[28] Рахимов А.T. // УФН. 2000. С. 996-999.

[29] Яфаров Р.К., Шалаев П.Д., Яфаров А.Р. // Радиотехника. 2016. № 7. C. 41-46.

[30] Whaley D.R. et al. // IEEE Trans. El. Dev. 2009. Vol. 56. N 5. P. 896-905.

[31] Whaley D.R., Duggal R., Armstrong C. et al. // IVEC-2013. DOI: 10.1109/IVEC.2013.6571009.

[32] Ulisse G., Brunetti F., Carlo A. // IVEC. 2010. P. 449-450.

[33] Gilchrist K.H., Piascik J.R., Stoner B.R., Radauscher E.J., Amsden J.J., Parker C.B., Glass J.T. // IVEC. 2014. P. 155156.

[34] Martin E.E., Trolan J.K., Dyke W.P. // J. Appl. Phys. 1960. Vol. 31. N 5. P. 782.

[35] Мелешкевич П.М. // Электронная техника. Сер. 1. СВЧтехника. 2016. Вып. 4 (531). С. 6-14.

[36] Бурцев А.А., Павлов А.А., Кищюк Е.П., Григорьев Ю.А., Данилушкин А.В., Шумихин К.В. // Письма в ЖТФ. 2017. Т. 43. Вып. 11. С. 89-94. 